\title{
Time Course of Antibiotic and Antifungal Concentrations in Corneal Organ Culture
}

\author{
Theo G. Seiler, MMed, * Markus Tschopp, MD, * Stefan Zimmerli, MD, $†$ Christoph Tappeiner, MD,* \\ Valéry V. Wittwer, MD, * and Beatrice E. Frueh, MD*
}

\begin{abstract}
Purpose: Contamination with bacteria and/or fungi is a serious complication in organ-cultured corneas. Hence, antibiotic and antifungal agents are added to the culture medium. The concentration of different antimicrobial and antifungal additives to the media over time has so far not been investigated in detail and is the aim of this study.

Methods: Nine human fresh corneoscleral discs were stored in corneal culture medium consisting of $2 \%$ fetal bovine serum and minimal essential medium. In addition, the culture medium contained $1200 \mu \mathrm{g} / \mathrm{mL}$ penicillin $\mathrm{G}, 25 \mu \mathrm{g} / \mathrm{mL}$ amphotericin $\mathrm{B}, 120$ $\mu \mathrm{g} / \mathrm{mL}$ streptomycin, and $100 \mu \mathrm{g} / \mathrm{mL}$ voriconazole. The concentration of amphotericin B used was 10 times higher than in clinical routine to facilitate its detection. The cultures were kept at $37^{\circ} \mathrm{C}$ for 28 days. At days $0,7,14,21$, and 28 , samples of the culture medium were harvested for analysis of antimicrobial concentrations by liquid chromatography and electrospray ionization tandem mass spectrometry.
\end{abstract}

Results: During corneal storage, the concentration of all antibiotics and antifungal agents declined significantly. By day 28, penicillin G was reduced to $14 \%$ of the original concentration. Amphotericin B and streptomycin retained approximately $60 \%$ of the original concentration to the end of the experiment and voriconazole maintained stable concentrations after an initial decline to approximately $80 \%$ at 7 days.

Conclusions: Throughout the entire storage period, the concentrations of penicillin G, streptomycin, and voriconazole exceeded the minimum inhibitory concentrations of all common contaminants, obviating the need for a change of the medium for antimicrobial reasons. Based on the minimum inhibitory concentrations and our findings, the initial concentration of amphotericin B should be raised to $5 \mu \mathrm{g} / \mathrm{mL}$.

Key Words: cornea, culture medium, concentration decay, antibiotics, antifungals, eye banking

(Cornea 2016;35:127-131)

S toring donor corneas is essential in modern eye banking. Many eye banks, especially in the United States, use cold storage at $4^{\circ} \mathrm{C}$ and commercially available solutions such as

Received for publication August 24, 2015; revision received September 15, 2015; accepted September 21, 2015. Published online ahead of print November 10, 2015

From the Departments of *Ophthalmology; and $\dagger$ Infectious Diseases, Inselspital, University of Bern, Bern, Switzerland.

The authors have no funding or conflicts of interest to disclose.

Reprints: Theo G. Seiler, MMed, Department of Ophthalmology, Inselspital, 3010 Bern, Switzerland (e-mail: theo@seiler.tv).

Copyright (C) 2015 Wolters Kluwer Health, Inc. All rights reserved.
Optisol (Bausch \& Lomb, Rochester, NY). This allows storage for up to 2 weeks. ${ }^{1,2}$

Warm preservation at 31 to $37^{\circ} \mathrm{C}$ is more commonly used in European eye banks: corneas can be stored up to 4 weeks in organ culture medium consisting of minimum essential medium with the addition of $2 \%$ fetal bovine serum. ${ }^{2-6}$ Antibiotics and antifungal agents are added to the organ culture media to kill contaminants and to avoid iatrogenic infections at transplantation. Despite this prophylaxis, severe complications like endophthalmitis can still occur. ${ }^{7,8}$ Little is known about the decay characteristics and efficacy over time of antimicrobial agents kept at body temperature. Therefore, we studied the concentration of the most commonly used antimicrobial and antifungal additives over time under the standard conditions established at the local eye bank at the Department of Ophthalmology, University Hospital in Bern.

\section{METHODS}

\section{Sample Preparation}

Nine human corneoscleral discs unsuitable for transplantation were excised under sterile conditions from intact globes not later than 6 hours post mortem and stored each in a corneal culture medium consisting of $2 \%$ fetal bovine serum and minimal essential medium (Sigma Aldrich, St Louis, MO) following the standard protocol of the eye bank of the University Hospital of Bern. The culture medium contained $1200 \mu \mathrm{g} / \mathrm{mL}$ penicillin $\mathrm{G}, 25 \mu \mathrm{g} / \mathrm{mL}$ amphotericin $\mathrm{B}, 120 \mu \mathrm{g} / \mathrm{mL}$ streptomycin, and $100 \mu \mathrm{g} / \mathrm{mL}$ voriconazole. The concentration of amphotericin B used in this study is 10 times higher than in clinical routine to allow easier detection. The corneoscleral discs were immersed each in $50 \mathrm{ml}$ of culture medium and were kept in screw-cap beakers at $37^{\circ} \mathrm{C}$ for 28 days.

At day $0,7,14,21$, and $28,1-\mathrm{mL}$ samples of culture media were aspirated under sterile conditions after stirring the culture media for 1 minute. The samples were sent to the laboratory (Interlabor, Belp, Switzerland) where they were stored at $4^{\circ} \mathrm{C}$ until analysis within 5 days.

\section{Measurement of Antimicrobial Concentrations}

Detection of penicillin G, amphotericin B, streptomycin, and voriconazole was based on liquid chromatography and electrospray ionization tandem mass spectrometry. 
Chromatographic separation was achieved at $25^{\circ} \mathrm{C}$ on a Phenomenex Gemini C-18 $5 \mu \mathrm{m}, 150-\times 2-\mathrm{mm}$ column using isocratic elution in a mobile phase of methanol and acetic acid in purified water. The samples were diluted with methanol and analyzed directly using a triple quadrupole mass spectrometer with positive electrospray ionization in multiple reaction monitoring mode. The limit of quantification of this procedure was $0.25 \mu \mathrm{g} / \mathrm{mL}$ for each compound.

\section{Statistical Analysis}

To compare the concentrations of the antibiotics at different times, the Wilcoxon test was used. Regression analysis of temporal decay of the concentrations was done assuming linear or exponential decay including the regression coefficient $\mathrm{R}^{2}$. All statistical calculations were performed with WinSTAT for Excel (R. Finch Software, 2015). $P<0.05$ was considered statistically significant.

\section{RESULTS}

\section{Absolute Concentrations}

During corneal storage, the concentration of antibiotics and antifungal substances declined significantly over time (penicillin G: $P=0.007$, after 7 days and later; amphotericin B: $P=0.03$, after 14 days and later; streptomycin: $P=0.008$, after 14 days and later; voriconazole: $P=0.008$, after 7 days). The absolute and percentage concentrations at different time points are listed in Tables 1 and 2 .

\section{Relative Concentrations and Decay Analysis}

Figure 1 shows the relative decrease of the concentration of penicillin $\mathrm{G}$ over time. The function was approximated by an exponential function $\mathrm{y}=0.892 \mathrm{e}^{-0.066 \mathrm{x}},\left(\mathrm{R}^{2}=0.9797\right)$ and a linear function $\mathrm{y}=-0.0293 \mathrm{x}+0.8646,\left(\mathrm{R}^{2}=0.8866\right)$ with $\mathrm{y}=$ relative concentration to the original concentration (microgram per milliliter); $\mathrm{x}=$ time (days). Obviously the exponential fit is superior to the linear one.

In Figure 2, the relative decrease of the concentration of amphotericin B over time is depicted. The function was approximated by an exponential function $\mathrm{y}=1.0134 \mathrm{e}^{-0.017 \mathrm{x}}$, $\left(\mathrm{R}^{2}=0.8815\right)$ and a linear function with $\mathrm{y}=-0.013 \mathrm{x}+$ $0.9969,\left(\mathrm{R}^{2}=0.9048\right)$ with $\mathrm{y}=$ relative concentration to the original concentration (microgram per milliliter); $\mathrm{x}=$ time (days). None of the 2 approximations seems to be superior.

The relative decrease of the streptomycin concentration over time is demonstrated in Figure 3. The function was approximated by an exponential function $\mathrm{y}=0.9762 \mathrm{e}^{-0.021 \mathrm{x}}$, $\left(\mathrm{R}^{2}=0.9179\right)$ and a linear function with $\mathrm{y}=-0.0161 \mathrm{x}+$ $0.9672,\left(R^{2}=0.9031\right)$ with $y=$ relative concentration to the original concentration (microgram per milliliter); $\mathrm{x}=$ time (days). None of the 2 approximations seems to be superior.

Decay of voriconazole includes an immediate reduction between day 0 and day 7 , from then on the concentration remains stable (Fig. 4). Neither linear nor exponential functions yield a meaningful mathematical fit (linear: $\mathrm{R}^{2}=$ 0.2949 , exponential: $\mathrm{R}^{2}=0.2771$ ). A 1 -step function is assumed.

\section{DISCUSSION}

Large studies in European eye banks have shown a contamination rate between $0.5 \%$ and $11 \%$ in organcultured corneas. ${ }^{9-15}$ The Hamburg eye bank found $4.7 \%$ fungal and $2.6 \%$ bacterial contaminants in 4546 tested cultures. ${ }^{9}$ In contrast, the Besançon group reported $1.1 \%$ fungal and $4.6 \%$ bacterial contamination in 1156 tested storage media. ${ }^{10}$ Variable culture protocols used in different eye banks may impact on the contamination spectra. For example, storage temperature at the Hamburg eye bank is $37^{\circ}$ $\mathrm{C}$, whereas at the Besançon eye bank it is $31^{\circ} \mathrm{C}$. Interestingly, the most common contaminating agents reported by both groups were similar: Staphylococcus spp., Streptococcus spp., Escherichia coli, and Candida spp. The antimicrobial agents tested in this study are active against all of these pathogens, and the concentrations used have been proven to be nontoxic for the corneal endothelium. ${ }^{9,16-18}$

Three different types of decay were identified: exponential - indicating a first-order chemical reaction, linearindicating a zero-order chemical reaction, and a 1-step decay. During the exponential decay (penicillin $G$ and possibly amphotericin B, and streptomycin), indicating a first-order chemical reaction, a constant percentage of penicillin $G$ is consumed per time unit. In processes with a linear decay (possibly amphotericin B, and streptomycin), the concentration is reduced by a constant amount per time unit. In exponential decay, the loss results from a simple degradation of the molecule itself and may be described by a half-time value. The linear decay may result from consumption by a constant number of corneal cells. The 1-step function is related to a single event (voriconazole) such as precipitation out of the solution or uptake by cells.

The European Committee on Antimicrobial Susceptibility Testing (EUCAST) published current results of the range of minimum inhibitory concentrations (MICs) for antimicrobial agents of a large European collection of bacterial and fungal

TABLE 1. Average Absolute Concentration and SD in Microgram Per Milliliter of Antibiotic and Antifungal Additives at Different Time Points

\begin{tabular}{lccccc}
\hline & Day 0 & Day 7 & Day 14 & Day 21 & Day 28 \\
\hline Penicillin G & $1124.0 \pm 272.3$ & $647.8 \pm 201.1$ & $360.9 \pm 105.2$ & $280.9 \pm 104.4$ & $162.9 \pm 65.9$ \\
Amphotericin B & $23.2 \pm 1.2$ & $19.9 \pm 3.2$ & $20.35 \pm 3.6$ & $17.7 \pm 3.3$ & $13.7 \pm 2.9$ \\
Streptomycin & $119.8 \pm 17.1$ & $102.5 \pm 20.9$ & $74.9 \pm 21.6$ & $76.7 \pm 29.0$ & $84.2 \pm 19.5$ \\
Voriconazole & $113.5 \pm 38.6$ & $82.7 \pm 8.6$ & $81.3 \pm 8.5$ & $87.5 \pm 13.7$ \\
\hline
\end{tabular}


TABLE 2. Averages Percentage of Concentration of Antibiotic and Antifungal Additives at Different Time Points

\begin{tabular}{lccccc}
\hline & $\begin{array}{c}\text { Day 0, } \\
\text { \% }\end{array}$ & $\begin{array}{c}\text { Day 7, } \\
\text { \% }\end{array}$ & $\begin{array}{c}\text { Day 14, } \\
\text { \% }\end{array}$ & $\begin{array}{c}\text { Day 21, } \\
\text { \% }\end{array}$ & $\begin{array}{c}\text { Day 28, } \\
\text { \% }\end{array}$ \\
\hline Penicillin G & 100 & 57 & 32 & 24 & 14 \\
Amphotericin B & 100 & 85 & 87 & 76 & 60 \\
Streptomycin & 100 & 87 & 64 & 65 & 55 \\
Voriconazole & 100 & 79 & 78 & 77 & 84 \\
\hline
\end{tabular}

pathogens. ${ }^{19}$ The MICs cited by the EUCAST for antibiotics and antifungals of the most common contaminating agents in corneal storage were used in this study.

Our results indicate that the penicillin G MICs for the most common gram-positive bacteria in corneal storage media, Staphylococcus and Streptococcus species, ${ }^{9,10}$ are exceeded for the total duration of the storage. The highest penicillin MIC currently reported by the EUCAST for coagulase-negative staphylococci and $S$. aureus is $128 \mu \mathrm{g} / \mathrm{mL} .{ }^{19}$ None of more than 37,000 isolates of Streptococcus pneumoniae had a penicillin MIC $>8 \mu \mathrm{g} / \mathrm{mL}^{19}$ In this study, the concentration of penicillin $\mathrm{G}$ never fell below the highest reported MIC for staphylococci and streptococci. Most probably, the decline in the concentration is not due to metabolism by cells but due to the degradation of penicillin G. In the literature, highly variable exponential decays are reported. ${ }^{20,21}$ Temperature is an important parameter: at $5^{\circ} \mathrm{C}$, the remaining concentration left after 1 month in bovine serum ${ }^{20}$ is approximately $25 \%$. In contrast, at $37^{\circ} \mathrm{C}$, a penicillin $\mathrm{G}$ half-life of 5 days was noted, ${ }^{21}$ which would result in a concentration of $25 \%$ after only 10 days. Our finding of a penicillin $\mathrm{G}$ concentration of $25 \%$ after approximately 19 days (Fig. 1) is within the reported range.

The reduction of both amphotericin B and streptomycin concentrations follows different kinetics (slow exponential or linear decay). The stability of streptomycin in Optisol was investigated at room temperature by Trousdale and colleagues ${ }^{17}$ who found no significant decrease in the concentration over a 4-week period, which is in accordance with data by industrial providers. ${ }^{22}$ The EUCAST reports streptomycin MICs in excess of $64 \mu \mathrm{g} / \mathrm{mL}$ for far less than $1 \%$ of $S$. aureus and coagulase-negative staphylococci. The streptomycin

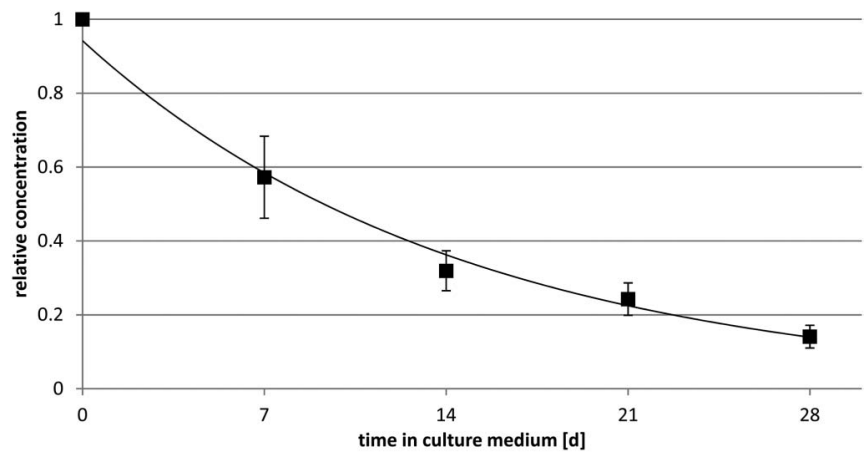

FIGURE 1. Relative concentration of penicillin $G$ at different time points $(d=$ days $)$ with $S D$ and exponential regression with $y=0.9762 e^{-0.021 x}$.

Copyright (C) 2015 Wolters Kluwer Health, Inc. All rights reserved.

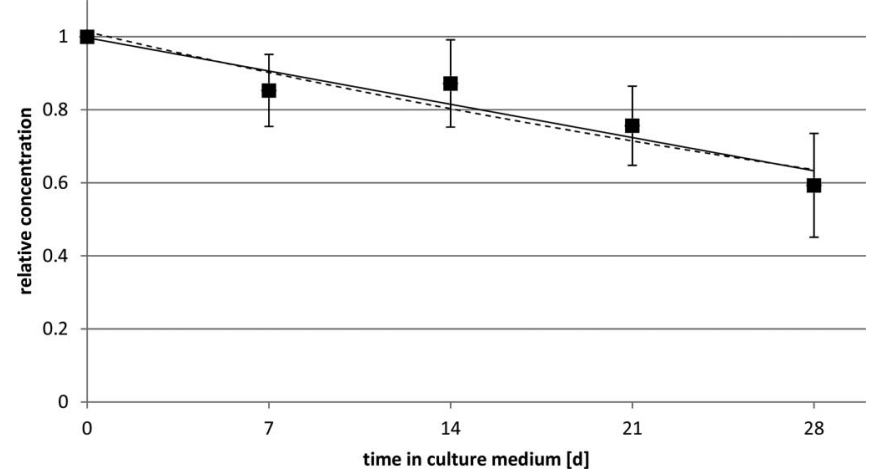

FIGURE 2. Relative concentration of amphotericin B at different time points $(d=$ days) with $S D$, dashed line shows exponential fit with $y=1.0134 \mathrm{e}^{-0.017 x}$, black line depicts the linear regression $y=-0.013 x+0.9969$.

levels found in our study inhibit more that $99 \%$ of staphylococcal isolates. However, $13 \%$ of $E$. coli isolates were reported to have streptomycin MICs $>64 \mu \mathrm{g} / \mathrm{mL}$ and might thus not be reliably inhibited by the concentrations achieved in our culture media. ${ }^{19}$ As resistance may become more frequent, corneal eye banks need to evaluate new antibiotics as additives to avoid contamination of the donor button.

The highest reported amphotericin B MIC is $2 \mu \mathrm{g} / \mathrm{mL}^{19}$ for the most common contaminating yeasts, which are Candida isolates (C. albicans, C. glabrata, and C. krusei). ${ }^{9,10}$ In Bern, like in other eye banks, ${ }^{9,14,15}$ amphotericin B is routinely used at $2.5 \mu \mathrm{g} / \mathrm{mL}$ as the initial concentration in the culture medium. Assuming an exponential decay, this would result in $1.5 \mu \mathrm{g} / \mathrm{mL}$ at the end of the storage period, which is below the highest MICs reported for Candida. If linear decay is assumed, like consumption by corneal cells, no amphotericin B should be left at day 28. Therefore, we recommend increasing the initial concentration to $5 \mu \mathrm{g} / \mathrm{mL}$, the highest concentration found to be nontoxic to endothelial cells. ${ }^{9}$ In Bern, where a medium change is performed for metabolic reasons at day 14 as suggested by Redbrake et $a{ }^{23}$ the amphotericin B concentration will exceed the highest MIC for Candida during the 14-day period of storage for both possible

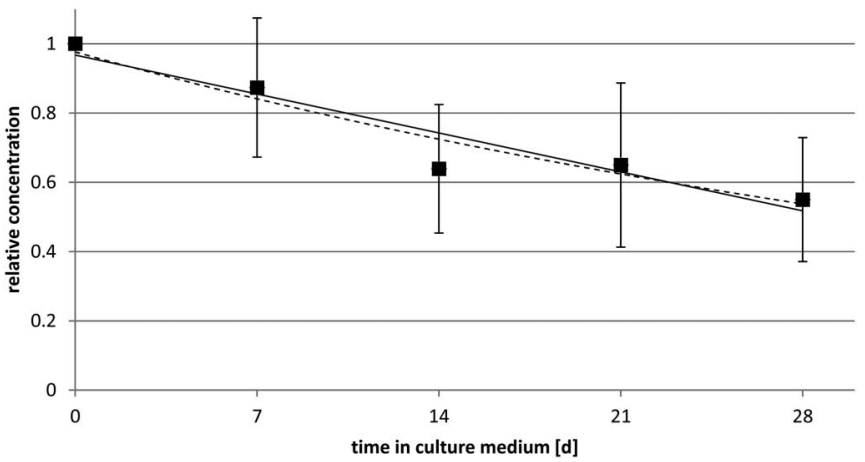

FIGURE 3. Relative concentration of streptomycin at different time points $(\mathrm{d}=$ days) with $\mathrm{SD}$, dashed line shows exponential fit with $y=0.9762 e^{-0.021 x}$, black line depicts the linear regression $y=-0.0161 x+0.9672$.

www.corneajrnl.com | 129 


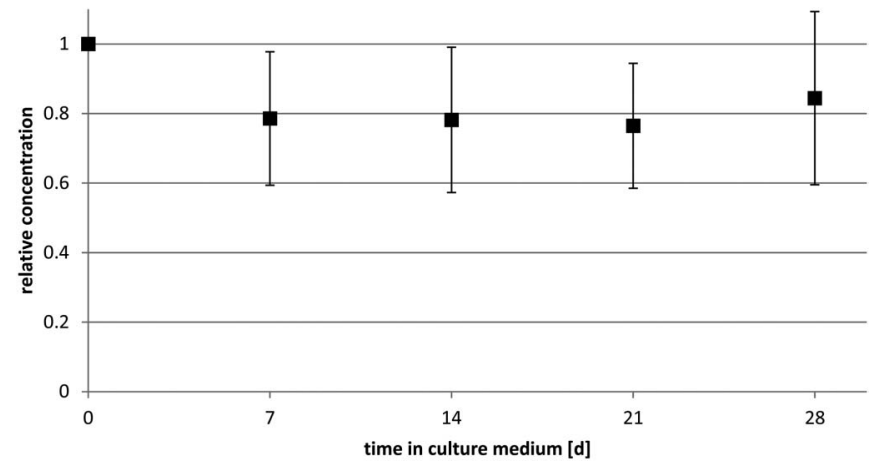

FIGURE 4. Relative concentration of voriconazol at different time points $(d=$ days $)$ with SD.

ways of decay. In other eye banks, for example in Bristol, ${ }^{11}$ $0.25 \mu \mathrm{g} / \mathrm{mL}$ of amphotericin is used routinely, without having higher reported fungal contamination rates. An explanation may be given by the EUCAST collecting Candida spp. in human infections and finding in approximately $70 \%$ of the cases sensitivity to amphotericin B concentrations of $0.25 \mu \mathrm{g} / \mathrm{mL}$. ${ }^{19}$

The voriconazole concentration does not show a constant decay, but a 1-step function. In all samples, we observed a loss from the starting point to day 7 and no further reduction thereafter. A possible explanation is precipitation out of the solution. The highest reported voriconazole $\mathrm{MIC}^{19,24}$ for $C$. glabrata, C. albicans, and C. krusei is less than $2 \mu \mathrm{g} / \mathrm{mL}$ and, therefore, the concentration is sufficiently high to inhibit fungal growth at all times up to day 28. Adams et $\mathrm{al}^{25}$ investigated the decay of voriconazole in polyvinyl chloride bags stored over 21 days at room temperature in a dark environment. They observed a linear reduction of approximately $15 \%$ and assumed precipitation on the surface of the polyvinyl chloride bag rather than decay. This is in accordance with our results.

Layer et $\mathrm{al}^{26}$ studied the efficacy of amphotericin B and voriconazole for controlling contamination in Optisol solutions. It is known that amphotericin B is a potent antifungal agent against Candida species and has no significant endothelial toxicity at concentrations below $5 \mu \mathrm{g} / \mathrm{mL} .^{9,26}$ Layer et al studied $C$. albicans and $C$. glabrata both of which were completely inhibited at an amphotericin B concentration of at least $4 \mu \mathrm{g} / \mathrm{mL}$. They also found that voriconazole at a concentration of $50 \mu \mathrm{g} / \mathrm{mL}$ compared with untreated controls did not result in a significant growth reduction. Voriconazole did not exhibit any endothelial toxicity up to $50 \mu \mathrm{g} / \mathrm{mL} .^{26}$

In contrast, Ritterband et $\mathrm{al}^{18}$ found a significant reduction in fungal contaminations in corneas stored in Optisol with voriconazole added. However, there were significant differences between both studies. ${ }^{18,26}$ Ritterband et al assessed corneal donor rims that had been stored for only 1 day, and performed cultures only if turbidity of the medium was noted. In contrast, Layer et al stored samples for 14 days and performed cultures on all media beakers. Furthermore, the concentration of voriconazole in the Ritterband group was 2 times higher $(100 \mu \mathrm{g} / \mathrm{mL})$ than the highest concentration used by the Layer group $(50 \mu \mathrm{g} / \mathrm{mL})$. Although it could be

\section{0 | www.corneajrnl.com}

argued that higher concentrations would be more effective, the concentrations used in Optisol should not exceed the published maximum MICs by a factor of 50 to 100 .

One limitation of this study is the long period from sample collection to analysis, which could not be reduced because of logistics.

In summary, amphotericin B and voriconazole seem to be comparable both for the spectrum of coverage of fungi important in eye banking and for the maintenance of therapeutic levels in organ culture media. There is no need to use both in the same medium. From an economic point of view, amphotericin $\mathrm{B}$ is cheaper and may, therefore, be preferable.

To conclude, current prevention of bacterial contamination with combined penicillin $\mathrm{G}$ and streptomycin — as used in most corneal storage media - seems to be sufficient. Only $E$. coli has a certain percentage $(13 \%)$ of resistant bacteria and, therefore, a new additive should be evaluated in the immediate future. The amphotericin B concentration should be raised to 5 $\mu \mathrm{g} / \mathrm{mL}$ to maintain effective concentrations over 4 weeks, especially when there is no media change during storage. Amphotericin B and voriconazole have equivalent effects on fungal contamination and, therefore, there is no need to use them simultaneously in the same medium.

\section{ACKNOWLEDGMENTS}

The authors thank Monika Kilchenmann for her work and support during the experiments.

\section{REFERENCES}

1. Lindstrom RL, Kaufman HE, Skelnik DL, et al. Optisol corneal storage medium. Am J Ophthalmol. 1992;114:345-356.

2. Frueh BE, Böhnke M. Prospective, randomized clinical evaluation of Optisol vs organ culture corneal storage media. Arch Ophthalmol. 2000; 118:757-760.

3. Frueh BE, Böhnke M. Corneal grafting of donor tissue preserved for longer than 4 weeks in organ-culture medium. Cornea. 1995;14: 463-466.

4. Doughman DJ, Harris JE, Schmitt MK. Penetrating keratoplasty using 37 C organ cultured cornea. Trans Sect Ophthalmol Am Acad Ophthalmol Otolaryngol. 1976;81:778-793.

5. Bigar F, Kaufman HE, McCarey BE, et al. Improved corneal storage for penetrating keratoplasties in man. Am J Ophthalmol. 1975;79:115-120.

6. Pels E, Schuchard Y. Organ-culture preservation of human corneas. Doc Ophthalmol. 1983;56:147-153.

7. Tappeiner C, Goldblum D, Zimmerli S, et al. Donor-to-host transmission of Candida glabrata to both recipients of corneal transplants from the same donor. Cornea. 2009;28:228-230.

8. Kloess PM, Stulting RD, Waring GO III, et al. Bacterial and fungal endophthalmitis after penetrating keratoplasty. Am J Ophthalmol. 1993; 115:309-316.

9. Linke SJ, Fricke OH, Eddy MT, et al. Risk factors for donor cornea contamination: retrospective analysis of 4546 procured corneas in a single eyebank. Cornea. 2013;32:141-148.

10. Khouani M, Debellemanière G, Malugani C, et al. Evaluation of microbial contamination of corneal transplants: one-year report from a French regional eye bank. Cornea. 2014;33:899-904.

11. Armitage WJ, Easty DL. Factors influencing the suitability of organcultured corneas for transplantation. Invest Ophthalmol Vis Sci. 1997;38: $16-24$.

12. Zanetti E, Bruni A, Mucignat G, et al. Bacterial contamination of human organ-cultured corneas. Cornea. 2005;24:603-607.

13. Patel HY, Brookes NH, Moffatt L, et al. The New Zealand National Eye Bank study 1991-2003: a review of the source and management of corneal tissue. Cornea. 2005;24:576-582.

Copyright (C) 2015 Wolters Kluwer Health, Inc. All rights reserved. 
14. Hagenah M, Böhnke M, Engelmann K, et al. Incidence of bacterial and fungal contamination of donor corneas preserved by organ culture. Cornea. 1995; 14:423-426.

15. Hermel M, Salla S, Hamsley N, et al. Detection of contamination during organ culture of the human cornea. Graefes Arch Clin Exp Ophthalmol. 2010;248:117-126

16. Hull DS, Green K, McQuaig CS, et al. Modification of the antibiotic system in M-K medium. Am J Ophthalmol. 1977;83:198-205.

17. Smith TM, Popplewell J, Nakamura T, et al. Efficacy and safety of gentamicin and streptomycin in Optisol-GS, a preservation medium for donor corneas. Cornea. 1995;14:49-55

18. Ritterband DC, Shah MK, Meskin SW, et al. Efficacy and safety of voriconazole as an additive in Optisol GS: a preservation medium for corneal donor tissue. Cornea. 2007;26:343-347.

19. European Committee on Antimicrobial Susceptibility Testing. Växjö, Sweden. Available at: http://www.eucast.org. Accessed August 18, 2015.

20. Heller DN, Smith ML, Chiesa OA. LC/MS/MS measurement of penicillin $\mathrm{G}$ in bovine plasma, urine, and biopsy samples taken from kidneys of standing animals. $J$ Chromatogr B Analyt Technol Biomed Life Sci. 2006;2:91-99.

21. Michnik A, Michalik K, Marcoin W. Influence of magnesium glutamate on stability of penicillin G aqueous solution. Int J Pharm. 2004;273:149-158.

22. Available at: https://www.applichem.com/de/shop/produktdetail/as/ streptomycinsulfat-ibiochemicai/. Accessed August 18, 2015.

23. Redbrake C, Salla S, Frantz A, et al. Metabolic changes of the human donor cornea during organ-culture. Acta Ophthalmol Scand. 1999;77: 266-272.

24. Marangon FB, Miller D, Giaconi JA, et al. In vitro investigation of voriconazole susceptibility for keratitis and endophthalmitis fungal pathogens. Am J Ophthalmol. 2004;137:820-825.

25. Adams AI, Morimoto LN, Meneghini LZ, et al. Treatment of invasive fungal infections: stability of voriconazole infusion solutions in PVC bags. Braz J Infect Dis. 2008;12:400-404.

26. Layer N, Cevallos V, Maxwell AJ, et al. Efficacy and safety of antifungal additives in Optisol-GS corneal storage medium. JAMA Ophthalmol. 2014;132:832-837. 